\title{
Recent Observations of PSR B1534+12
}

\section{H. Stairs}

University of Manchester, Jodrell Bank Observatory, Macclesfield, Cheshire SK11 9DL U.K.

S. E. Thorsett

Department of Astronomy and Astrophysics, University of California, 1156 High St., Santa Cruz, CA 95064 USA

J. H. Taylor

Joseph Henry Laboratories and Physics Department, Princeton University, Princeton, NJ 08544 USA

\section{Z. Arzoumanian}

NASA Goddard Space Flight Center, Mailstop 662.0, Greenbelt, MD 20771 USA

\begin{abstract}
We present the results of recent Arecibo observations of the relativistic double-neutron-star binary PSR B1534+12. The timing solution includes measurements of five post-Keplerian orbital parameters, whose values agree well with the predictions of general relativity. The observations show that the pulse profile is evolving secularly at both 1400 $\mathrm{MHz}$ and $430 \mathrm{MHz}$. This effect is similar to that seen in PSR B1913+16, and is almost certainly due to general relativistic precession of the pulsar's spin axis. We also present high-quality polarimetric profiles at both observing frequencies.
\end{abstract}

The strong-field regime of gravity is best tested through timing observations of double-neutron-star binary pulsars in close, highly eccentric orbits. Observations of PSR B1913+16 have allowed the measurement of three "postKeplerian" (PK) parameters: the rate of periastron advance, $\dot{\omega}$, the time-dilation and gravitational-redshift parameter, $\gamma$, and the rate of orbital period decay, $\dot{P}_{b}$ (Taylor \& Weisberg 1989). These parameters agree with the predictions of general relativity (GR) to better than $1 \%$.

We have observed the similar system PSR B1534+12 between 1990 and 2000 , primarily with the $305 \mathrm{~m}$ Arecibo telescope, but also with the $43 \mathrm{~m}$ telescope at Green Bank, W.V., and the $76 \mathrm{~m}$ Lovell Telescope at Jodrell Bank, U.K. For this pulsar, an additional two PK parameters are measurable: the Shapiro-delay parameters, $r$ and $s$ (Stairs et al. 1998). These observations provide a $1 \%$ test of the purely non-radiative sector of GR, complementing the test provided by PSR B1913+16. Furthermore, under the assumption that GR is the correct theory of gravity, we are able to derive the distance to the pulsar based on the required kinematic correction to $\dot{P}_{b} ;$ we find a distance of $1.08 \pm 0.15 \mathrm{kpc}$, 

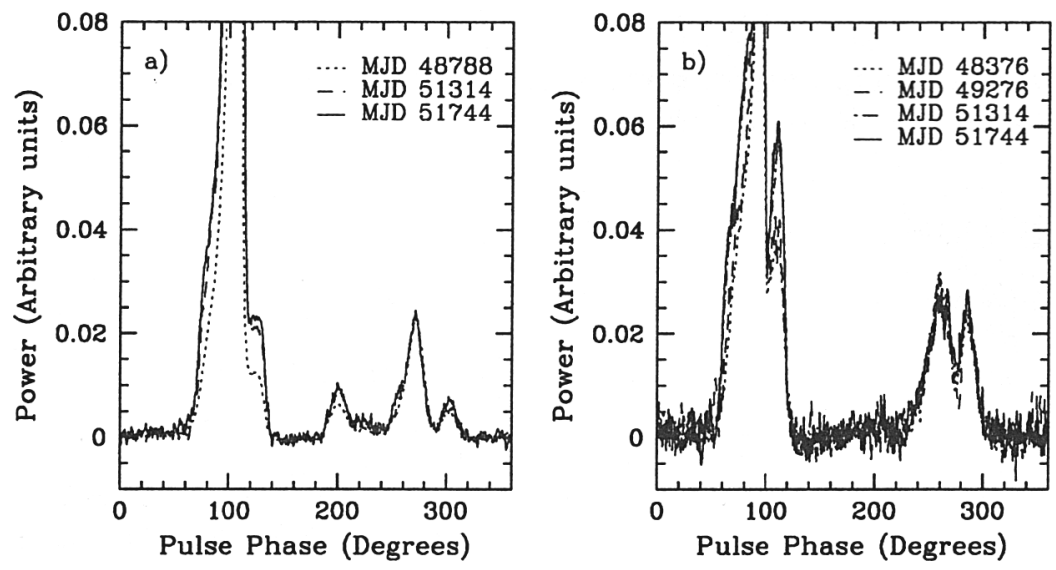

Figure 1. Evolution of the pulse profile of PSR B1534+12 with time, using the interpulse as a fiducial reference, at a) $430 \mathrm{MHz}$ and b) $1400 \mathrm{MHz}$.

somewhat larger than the $0.7 \pm 0.2 \mathrm{kpc}$ predicted from the pulsar's dispersion measure (Taylor \& Cordes 1993).

A further prediction of GR is geodetic precession. The recycled pulsar's spin axis and the orbital angular momentum vector are expected to be misaligned on evolutionary grounds. Their resultant coupling will cause the spin axis to precess at a measurable rate. This precession results in a change in the line-of-sight cut across the emission region of the pulsar, and hence in a secularly changing pulse profile. Evidence for this effect has been seen in PSR B1913+16 (e.g, Weisberg, Romani \& Taylor 1989; Kramer 1998; Kramer et al, these proceedings). Similar profile shape changes in PSR B1534+12 at $1400 \mathrm{MHz}$ were noted by Arzoumanian (1995) in examining Arecibo observations from 1990-94. Our recent data confirm Arzoumanian's observations of precession, and reveal similar evolution in the pulse shape at $430 \mathrm{MHz}$. This can be seen in Figure 1, in which the small interpulse is taken as a reference point at both frequencies. Our eventual goal is to combine the information derived from the pulsar's polarization properties with a model of the pulsar emission region to completely determine the geometry of the system and perhaps test the rate at which precession is occurring.

\section{References}

Arzoumanian, Z. 1995, $\mathrm{PhD}$ thesis, Princeton University

Kramer, M. 1998, ApJ, 509, 856

Stairs, I. H. et al. 1998, ApJ, 505, 352

Taylor, J. H. \& Weisberg, J. M. 1989, ApJ, 345, 434

Taylor, J. H. \& Cordes, J. M. 1993, ApJ, 411, 674

Weisberg, J. M., Romani, R. W., \& Taylor, J. H. 1989, ApJ, 347, 1030 